\title{
Behavioural inhibition and behavioural activation system scales for children: relationships with Eysenck's personality traits and psychopathological symptoms
}

\author{
Peter Muris $^{\mathrm{a}, *}$, Cor Meesters ${ }^{\mathrm{b}}$, Elske de Kanter ${ }^{\mathrm{b}}$, Petra Eek Timmerman ${ }^{\mathrm{b}}$ \\ ${ }^{a}$ Institute of Psychology, Erasmus University Rotterdam, Burgemeester Oudlaan 50, Suite J5-31, \\ P.O. Box 1738, 3000 DR Rotterdam, The Netherlands \\ ${ }^{\mathrm{b}}$ Department of Medical, Clinical, and Experimental Psychology, Maastricht University, P.O. Box 616, \\ 6200 MD Maastricht, The Netherlands
}

Received 8 December 2003; received in revised form 14 May 2004; accepted 8 June 2004 Available online 10 August 2004

\begin{abstract}
This study examined the psychometric properties of an age-downward version of the Carver and White (1994) BIS/BAS scales. Normal school children $(N=284)$ aged $8-12$ years completed the BIS/BAS scales as well as scales of Neuroticism, Extraversion, and psychopathological symptoms. Results showed that the BIS/BAS scales for children had a theoretically meaningful factor structure and satisfactory reliability. Relationships with Eysenck's personality traits of Neuroticism and Extraversion, and psychopathological symptoms were generally as expected. That is, Neuroticism was positively associated with both BIS and BAS, whereas Extraversion was negatively related to BIS but positively to BAS. Further, BIS was convincingly connected to higher levels of internalizing symptoms, whereas BAS was to a certain level related to externalizing symptoms.
\end{abstract}

(C) 2004 Elsevier Ltd. All rights reserved.

Keywords: Behavioural inhibition; Behavioural activation; Children; Personality; Psychopathology

\footnotetext{
* Corresponding author. Tel.: +31 10408 8706; fax: +31 104089009.

E-mail address: muris@fsw.eur.nl (P. Muris).
} 


\section{Introduction}

The biological personality theory of Gray $(1987,1991)$ posits two main brain systems that are hypothesized to regulate approach and withdrawal behaviour in response to environmental stimuli: the behavioural inhibition system (BIS) and the behavioural activation system (BAS). The BIS serves to alert the person to the possibility of danger or punishment, thereby enhancing avoidance behaviour. Activity in the BIS is responsible for feelings of anxiety and incites the individual to stop whatever action is going on and to scan the environment for further cues. The BAS is sensitive to signals of reward, and involved in approach behaviour. Activity in the BAS produces impulsive behaviour: the person will vigorously pursue any action that might result in reward, with little attention to the possibility of negative consequences.

According to Gray, individual differences in BIS and BAS activity underlie the supertraits of neuroticism and extraversion that are central to Eysenck's (1967) theory of personality. The supertrait of neuroticism refers to the ease and frequency with which the person becomes upset and distressed, whereas the supertrait of extraversion concerns tendencies towards sociability, craving for excitement, liveliness, activeness, and dominance. Gray has argued that neuroticism reflects a summation of the activities in BIS and BAS: neurotic people are those whose BIS and BAS are both highly reactive. Gray assumes that extraversion reflects a combination of high BAS and low BIS activity: extraverted people are more responsive to positive than to negative outcomes and consequences. Although Gray's point of view is not entirely supported by empirical data, there is indeed evidence showing that high BIS activity is associated with neuroticism, whereas high BAS activity is closely related to extraversion (Carver \& White, 1994; Caseras, Avila, \& Torrubia, 2003; Heubeck, Wilkinson, \& Cologon, 1998; Jorm et al., 1999).

It has been theorized that various types of psychopathology can be explained by different constellations of BIS and BAS. For instance, high levels of BIS activity are thought to be linked to anxiety symptoms (Gray, 1982), whereas low levels of BIS activity have been proposed to be associated with attention-deficit and hyperactivity disorder (Quay, 1988) and psychopathy (Fowles, 1980). In addition, high levels of BAS activity are hypothesized to be related to conduct disorder and antisocial personality disorder (Quay, 1993), whereas low levels of BAS activity have been regarded as a marker for depression (Depue, Krauss, \& Spoont, 1987). Considerable evidence has been obtained in recent years for the supposed connections between levels of BIS and BAS activity, on the one hand, and specific forms of psychopathology, on the other hand (see for a comprehensive review, Johnson, Turner, \& Iwata, 2003).

Whereas the concepts of BIS and BAS are also considered to be relevant for child psychopathology, relatively few studies have examined these two brain systems in youths. Most of this research has employed laboratory tasks to assess reactions to stop signals and punishment and reward contingencies for measuring levels of BIS and BAS in clinically referred children (Casey, Castellanos, Giedd, \& Marsh, 1997; Matthys, Van Goozen, De Vries, Cohen-Kettenis, \& Van Engeland, 1998; Scheres, Oosterlaan, \& Sergeant, 2001). While such tasks provide important information on BIS and BAS activity of youths suffering from a psychiatric disorder (in particular Attention-Deficit and Hyperactivity Disorder), relatively little is known about these motivational brain systems in normal children. This is at least partly due to the fact that there is currently no easy-to-administer instrument for assessing BIS and BAS in young people. A self- 
report questionnaire for measuring dispositional BIS and BAS sensitivities in children would certainly be a valuable addition to the literature, and might promote further research on these biologically-based personality dimensions in youth populations. With this in mind, the current study examined the psychometric properties of an age-downward version of the Carver and White (1994) BIS/BAS scales. A sample of normal school children $(N=284)$ aged $8-12$ years completed the BIS-BAS scales as well as a set of questionnaires measuring Eysenck's personality traits of Neuroticism and Extraversion and psychopathological symptoms. Furthermore, parents also filled out questionnaires assessing psychopathological symptoms in their children. In this way, it became possible to examine (a) the factor structure of the BIS/BAS scales for children, (b) the reliability of the BIS/BAS scales, (c) relationships between BIS/BAS and Eysenck's personality traits of neuroticism and extraversion, and (d) relationships between BIS/BAS and selfand parent-reported child psychopathology.

\section{Method}

\subsection{Participants and procedure}

Participants were 284 children (136 boys and 148 girls) recruited from regular primary schools in Goirle and Maastricht, The Netherlands. Mean age of the children was 10.07 years ( $\mathrm{SD}=0.96$, range 8-12 years). Most children (i.e., $>90 \%$ ) were Caucasian. Parent questionnaires were completed by mother $(69.7 \%)$, father $(9.9 \%)$, both parents $(19.4 \%)$, or another person who raised the child $(1.0 \%)$.

Parents and children $(N=565)$ received a letter, which provided them with information about the study and invited them to participate. Three hundred parents and children (53.1\%) responded favourably to this invitation. Parents completed the set of questionnaires, and returned the materials to the researchers by mail. Children were tested during a classroom session at school. During this session, the teacher and a research assistant were always available to ensure confidential and independent responding and to provide assistance when necessary. Due to missing data, the final sample consisted of 284 children and their parents (i.e., 50.3\% of those initially invited to participate).

\subsection{Questionnaires}

\subsubsection{Child report}

The BIS/BAS scales (Carver \& White, 1994) have been constructed to assess individual differences in sensitivity of the BIS and BAS. The original items were somewhat changed and simplified to make them more appropriate and understandable to children. Each item had to be scored on a four-point Likert scale with $0=$ not true, $1=$ somewhat true, $2=$ true, and $3=$ very true. Previous factor analytic studies using the BIS/BAS scales in adults (e.g., Carver \& White, 1994) have consistently demonstrated that the 20 items can be allocated to four subscales: BIS, BAS Reward responsiveness, BAS Drive, and BAS Fun seeking (see Table 1).

The Junior version of the Eysenck Personality Questionnaire (JEPQ; Eysenck \& Eysenck, 1975) was employed to assess Neuroticism and Extraversion. Items are dichotomous and have to be 
Table 1

Two-factor solution of the BIS/BAS scales for children as obtained with principal component analysis

\begin{tabular}{|c|c|c|c|c|}
\hline & \multicolumn{2}{|c|}{$\begin{array}{l}\text { Oblique } \\
\text { rotation }\end{array}$} & \multicolumn{2}{|c|}{$\begin{array}{l}\text { Varimax } \\
\text { rotation }\end{array}$} \\
\hline & 1 & 2 & 1 & 2 \\
\hline \multicolumn{5}{|l|}{$B I S$} \\
\hline I usually get very tense when I think something unpleasant is going to happen & 0.74 & 0.19 & 0.74 & 0.01 \\
\hline I worry about making mistakes & 0.81 & 0.15 & 0.81 & 0.00 \\
\hline I am hurt when people scold me or tell me that I do something wrong & 0.50 & 0.50 & 0.46 & 0.44 \\
\hline I feel pretty upset when I think that someone is angry with me & 0.71 & 0.30 & 0.70 & 0.21 \\
\hline I do not become fearful or nervous, even when something bad happens to me (R) & -0.38 & 0.12 & -0.39 & 0.17 \\
\hline I feel worried when I think I have done poorly at something & 0.79 & 0.20 & 0.79 & 0.01 \\
\hline I am very fearful compared to my friends & 0.58 & 0.16 & 0.58 & 0.01 \\
\hline \multicolumn{5}{|l|}{ BAS reward responsiveness } \\
\hline I feel excited and full of energy when I get something that I want & 0.15 & 0.58 & 0.11 & 0.57 \\
\hline When I am doing well at something, I like to keep doing this & -0.07 & 0.31 & -0.01 & 0.33 \\
\hline I get thrilled when good things happen to me & 0.30 & 0.54 & 0.26 & 0.51 \\
\hline I get very excited when I would win a contest & 0.28 & 0.52 & 0.24 & 0.50 \\
\hline I get really excited when I see an opportunity to get something I like & 0.21 & 0.72 & 0.16 & 0.70 \\
\hline \multicolumn{5}{|l|}{ BAS drive } \\
\hline When I want something, I usually go all the way to get it & 0.18 & 0.73 & 0.13 & 0.72 \\
\hline I do everything to get the things that I want & 0.24 & 0.69 & 0.19 & 0.67 \\
\hline When I see an opportunity to get something that I want, I go for it right away & 0.09 & 0.71 & 0.00 & 0.71 \\
\hline Nobody can stop me when I want something & 0.21 & 0.64 & 0.17 & 0.62 \\
\hline \multicolumn{5}{|l|}{ BAS fun seeking } \\
\hline I often do things for no other reason than that they might be fun & 0.23 & 0.36 & 0.20 & 0.34 \\
\hline I crave for excitement and new sensations & 0.05 & 0.55 & 0.00 & 0.55 \\
\hline I am always willing to try something new, when I think it will be fun & -0.08 & 0.39 & -0.12 & 0.41 \\
\hline I often do things on the spur of the moment & 0.34 & 0.24 & 0.33 & 0.20 \\
\hline
\end{tabular}

Notes. $\mathrm{BIS}=$ behavioural inhibition system, $\mathrm{BAS}=$ behavioural activation system, $\mathrm{R}=$ reversed item. For each item, the highest factor loading is printed in bold.

answered by yes or no. After recoding inversely formulated items, a total score can be computed for each personality trait by summing across relevant items. The psychometric properties of the JEPQ are satisfactory (e.g., Eysenck, Von Knorring, \& Von Knorring, 1988).

The self-report version of the Strengths and Difficulties Questionnaire (SDQ-C; Goodman, 2001) consists of 25 items describing positive and negative attributes of children. Items have to be scored on a 3 -point scale with $0=$ not true, $1=$ somewhat true, and $2=$ certainly true. In the current study, the following scores were computed by summing scores on relevant items (after recoding reversed items): Emotional problems, Hyperactivity/Conduct problems, and Prosocial behaviour. Research has shown that the SDQ has good psychometric properties (Goodman, 2001).

The short version of the Revised Child Anxiety and Depression Scale (RCADS; Chorpita, Yim, Moffitt, Umemoto, \& Francis, 2000) is a 25-item scale for measuring symptoms of DSM-defined 
anxiety disorders and depression. Items have to be scored on a 4-point scale with $0=$ never, $1=$ sometimes, 2 often, and $3=$ always. In the present study, an Anxiety and a Depression score were obtained by summing across relevant items. Previous research has indicated that the (shortened) RCADS has a clear-cut factor structure, is reliable in terms of internal consistency and temporal stability, and displays good validity as evidenced by positive associations with concurrent childhood questionnaires (Muris, Meesters, \& Schouten, 2002).

The Child Rating of Aggression (CRA) is a self-report version of the Teacher Rating Scale for Reactive and Proactive Aggression (Brown, Atkins, Osborne, \& Milnamow, 1996), a 21-item questionnaire for assessing antisocial behaviours in children and adolescents. Each item has to be scored on a 5 -point scale with $0=$ never, $1=$ seldom, $2=$ sometimes, $3=$ often, and $4=$ almost always. A total Aggression score was computed by summing scores to all items. There is clear support for the psychometric qualities of the TRA. That is, the scale is reliable and satisfactorily discriminates between non-aggressive and aggressive children (Dodge, Lochman, Harnish, Bates, \& Pettit, 1997).

\subsubsection{Parent report}

Parent versions of the SDQ-C (i.e., SDQ-P), RCADS (i.e., RCADS-P), and CRA (i.e., PRA) were used to assess children's levels of Emotional problems, Hyperactivity/Conduct problems, Prosocial behaviour, Anxiety, Depression, and Aggression from the parents' point of view.

\section{Results}

\subsection{Factor structure of BIS/BAS scales for children}

Following the procedure employed in previous research (e.g., Carver \& White, 1994), a principal components factor analysis with Oblique rotation was carried out to examine the structure of the BIS/BAS scales. This analysis showed that the first four-factors indeed had eigenvalues greater than 1 , and together accounted for $50.00 \%$ of the variance. Inspection of the four-factor structure, however, revealed that this solution was not satisfactory. Although some support emerged for three of the original factors, namely BIS, BAS Drive, and BAS Reward responsiveness, the fourth factor only consisted of one reversely scored BIS item (i.e., "I do not become fearful or nervous, even when something bad happens to me") whereas BAS Fun seeking items did not appear as a separate factor.

An additional factor analysis was performed to test the two-factor solution of the BIS/BAS scales. The two-factors accounted for $38.09 \%$ of the total variance, and were only marginally correlated $(r=0.21, p<0.001)$. As can be seen in Table 1, one factor consisted of BIS items, whereas the other factor contained BAS items. Only two items did not correspond with this theoretically meaningful pattern. That is, the BIS item "I am hurt when people scold me or tell me that I do something wrong" loaded high on both factors, whereas the BAS Fun seeking item "I often do things on the spur of the moment" loaded more substantially on the BIS than on the BAS factor. According to Gray's $(1987,1991)$ original model, a two-factor orthogonal structure of the BIS/ BAS scales should be expected. To test this model, the factor analysis was repeated, this time employing a Varimax rotation. As shown in Table 1, this analysis yielded a highly similar pattern 
of results. Altogether, it seemed most appropriate to conclude that the BIS/BAS scales for children contain two-factors, namely BIS and BAS, and these will be the focus of our analyses in the further course of this article.

\subsection{Reliability of the BIS/BAS scales for children}

The BIS/BAS scales appeared to be reliable in terms of internal consistency: Cronbach's alphas were 0.78 for BIS and 0.81 for BAS. Note further that most other questionnaires that were employed in the current study also had acceptable reliability coefficients (see Table 2). The only exceptions were the Prosocial behaviour subscale of the SDQ-C and the Depression scales of the RCADS and RCADS-P, which had alphas of lower than 0.60.

As an aside, it should be mentioned that there were very few significant gender differences in this sample. Boys scored higher on Hyperactivity/Conduct problems as rated by parents, means being $4.54(\mathrm{SD}=3.63)$ versus $3.20(\mathrm{SD}=2.80)$, respectively $[t(282)=3.52, p<0.01]$, whereas girls displayed higher levels of Prosocial behaviour, and this was true for child- as well as parentreport, means being $8.08(\mathrm{SD}=1.47)$ versus $7.10(\mathrm{SD}=1.76)$ and $8.79(\mathrm{SD}=1.46)$ versus 8.19 $(\mathrm{SD}=2.02)$ [ts being 5.11 and 2.88 , respectively, both $p \mathrm{~s}<0.01]$.

\subsection{Relationships between BIS/BAS and personality traits/psychopathological symptoms}

Table 2 presents Pearson correlations between BIS/BAS, on the one hand, and Eysenck's personality traits and psychopathological symptoms, on the other hand. As there was a significant correlation between BIS and BAS $(r=0.38, p<0.001)$, we decided to control for this shared variance by computing partial correlations. Thus, correlations between BIS and personality traits/ psychopathological symptoms were computed while controlling for BAS scores, whereas correlations between BAS scales and personality traits/psychopathological symptoms were calculated while holding BIS levels constant.

Results showed that the correlations between BIS/BAS and the personality traits of Neuroticism and Extraversion showed the to be expected pattern. More precisely, Neuroticism was positively associated with both BIS and BAS ( $r$ s being 0.67 and 0.17 , respectively, both $p \mathrm{~s}<0.01$ ), with the former correlation being significantly larger than the latter correlation $(Z=8.83$, $p<0.001)$. Extraversion was negatively related to BIS $(r=-0.34, p<0.001)$ but positively to BAS $(r=0.19, p<0.01)$, although the latter link was less substantial than expected.

BIS appeared connected to a broad range of child-reported psychopathological symptoms ( $r$ s between 0.28 and 0.63 , all $p s<0.001$ ). Particularly strong correlations emerged between BIS and SDQ-C Emotional problems and RCADS Anxiety symptoms. BAS was less convincingly related to psychopathology: only small correlations with SDQ-C Hyperactivity/ Conduct problems and CRA Aggression emerged ( $r$ s being 0.16 and 0.13 , respectively, $p \mathrm{~s}<0.05$ ).

Correlations between BIS/BAS and children's psychopathological symptoms as rated by parents confirmed some of the links that were found between BIS/BAS and child-reported symptoms. That is, BIS appeared significantly connected to SDQ-P Emotional problems and RCADS-P Anxiety and Depression $(r$ between 0.15 and $0.39, p s<0.05)$, whereas BAS was only related to SDQP Hyperactivity/Conduct problems $(r=0.21, p<0.05)$. 
Table 2

Descriptive statistics (means, standard deviations, and reliability coefficients) for all questionnaires, and correlations between BIS/BAS scales and other measures

\begin{tabular}{|c|c|c|c|c|}
\hline & Total group $(N=284)$ & $\alpha$ & $\mathrm{BIS}^{\mathrm{a}}$ & $\mathrm{BAS}^{\mathrm{b}}$ \\
\hline \multicolumn{5}{|l|}{ Child report } \\
\hline \multicolumn{5}{|l|}{ BIS/BAS scales } \\
\hline BIS & $6.90(3.87)$ & 0.78 & & \\
\hline BAS & $16.85(6.39)$ & 0.81 & $0.38^{* * *}$ & \\
\hline \multicolumn{5}{|l|}{ JEPQ } \\
\hline Neuroticism & $5.76(4.37)$ & 0.84 & $0.67^{* * *}$ & $0.17^{* *}$ \\
\hline Extraversion & $16.32(3.36)$ & 0.71 & $-0.34^{* * *}$ & $0.19^{* *}$ \\
\hline \multicolumn{5}{|l|}{ SDQ-C } \\
\hline Emotional problems & $2.31(1.97)$ & 0.64 & $0.63^{* * *}$ & 0.01 \\
\hline Hyperactivity/conduct problems & $5.64(2.74)$ & 0.61 & $0.28^{* * *}$ & $0.16^{* *}$ \\
\hline Prosocial behaviour & $7.61(1.68)$ & 0.56 & -0.06 & 0.01 \\
\hline \multicolumn{5}{|l|}{ RCADS } \\
\hline Anxiety & $10.04(6.28)$ & 0.84 & $0.58^{* * *}$ & 0.10 \\
\hline Depression & $2.54(1.61)$ & 0.49 & $0.29^{* * *}$ & 0.03 \\
\hline \multicolumn{5}{|l|}{ CRA } \\
\hline Aggression & $8.64(5.13)$ & 0.79 & $0.29^{* * *}$ & $0.13^{*}$ \\
\hline \multicolumn{5}{|l|}{ Parent report } \\
\hline \multicolumn{5}{|l|}{ SDQ-P } \\
\hline Emotional problems & $2.12(2.18)$ & 0.75 & $0.27^{* * *}$ & -0.03 \\
\hline Hyperactivity/conduct problems & $3.84(3.29)$ & 0.78 & 0.08 & $0.21^{* * *}$ \\
\hline Prosocial behaviour & $8.50(1.77)$ & 0.72 & 0.01 & -0.07 \\
\hline \multicolumn{5}{|l|}{ RCADS-P } \\
\hline Anxiety & $7.79(5.26)$ & 0.84 & $0.39^{* * *}$ & -0.07 \\
\hline Depression & $1.45(1.36)$ & 0.49 & $0.15^{*}$ & -0.01 \\
\hline \multicolumn{5}{|l|}{ PRA } \\
\hline Aggression & $13.64(7.62)$ & 0.90 & 0.09 & 0.10 \\
\hline
\end{tabular}

Notes. $\mathrm{BIS}=$ behavioural inhibition system, $\mathrm{BAS}=$ behavioural activation system, JEPQ $=$ junior version of the Eysenck personality questionnaire, SDQ-C $=$ child version of the strengths and difficulties questionnaire, $\mathrm{RCADS}=$ revised child anxiety and depression scale, $\mathrm{CRA}=$ child rating of aggression, SDQ-P = parent version of the strengths and difficulties questionnaire, RCADS-P = parent version of the revised child anxiety and depression scale, $\mathrm{PRA}=$ parent rating of aggression.

${ }^{*} p<0.05$.

${ }^{* *} p<0.01$.

$p<0.001$.

${ }^{a}$ Correlations corrected for BAS.

${ }^{\mathrm{b}}$ Correlations corrected for BIS. 


\section{Discussion}

The current study examined the psychometric properties of a child version of the Carver and White (1994) BIS/BAS scales. The main results can be summarized as follows. First of all, although the hypothesized four-factor structure of the BIS/BAS scales was not found in this sample of normal children, a theoretically meaningful solution was found with two separate factors for BIS and BAS. Second, the reliability of the BIS and BAS scales appeared satisfactory with Cronbach's alphas of, respectively, 0.78 and 0.81 . Third, the BIS/BAS scales generally showed the expected relationships with Eysenck's personality traits of Neuroticism and Extraversion and psychopathological symptoms.

Although these results provide some support for the psychometric qualities of the BIS/BAS scales for children, there were a number of less favourable findings, which deserve some further comment. To begin with, a modest but significant, positive correlation was found between BIS and BAS. This is in disaccord with previous research in adults showing that BIS is not associated with BAS in general, but only to some extent with BAS Reward responsiveness (e.g., Jorm et al., 1999; Ross, Millis, Bonebright, \& Bailley, 2002). It is possible that this result has to do with children's greater susceptibility to response tendency, that is the inclination to score consistently lower or higher on questionnaire items. Further, the factor analysis of the BIS/BAS scales did not yield the hypothesized four-factor solution (Carver \& White, 1994; Franken, Muris, \& Rassin, in press; Heubeck et al., 1998; Jorm et al., 1999; Leone, Perugini, Bagozzi, Pierro, \& Mannetti, 2001). While spurs of the factors BAS Reward responsiveness and BAS Drive were found, there were in particular problems with BAS Fun seeking, which did not emerge as a separate factor. There are several explanations for this unexpected finding. First, it should be mentioned that the BIS/ BAS scales were adapted for use with children, and although the researchers were well acquainted with Gray's BIS/BAS model and the original Carver and White scales, the possibility cannot be ruled out that this procedure had a negative impact on the structure of the scale. Second, it is important to note that although the Carver and White (1994) BIS/BAS scales are generally regarded as a good instrument for assessing Gray's basic personality dimensions, it is also true that there is still debate on the subdivision of the BAS dimension. For example, Caseras et al. (2003) who studied the factor structure of various BAS-related scales, come to the conclusion that BAS consists of two-factors, namely "reward interest" and "impulsiveness-thrill seeking". Third, one should keep in mind that the children in the present study were still relatively young. Even though it can be assumed that BAS is biologically determined, it may well be that environmental influences (e.g., parents providing extremely high or low levels of reward) or differences in the maturation of regulative temperament traits play an important role in the manifestation of this motivational brain system (e.g., Nigg, 2000).

The relationships of BIS/BAS with personality traits were as predicted. For example, Neuroticism was positively associated with both BIS and BAS, whereas Extraversion was negatively related to BIS but positively to BAS. Admittedly, the correlation between BAS and extraversion was less substantial than expected (see Carver \& White, 1994; Jorm et al., 1999), a finding that is in keeping with the notion that BAS is less clearly manifest in young people. As to their links with symptoms of psychopathology, it was found that BIS was convincingly connected to higher levels of internalizing symptoms (i.e., Emotional problems, Anxiety, and Depression), whereas BAS was to a certain extent related to externalizing symptoms (e.g., Hyperactivity/Conduct prob- 
lems and Aggression). With regard to the link between BIS/BAS and psychopathology, three additional remarks are in order. First of all, one could argue that the convincing correlations between BIS and internalizing symptoms were partly due to measurement confounding (i.e., item overlap; for a comprehensive discussion, see Lemery, Essex, \& Smider, 2002). While it is certainly true that the BIS scale and questionnaires for assessing internalizing symptoms are closely allied, it seems important to keep in mind that BIS items are worded to reflect a propensity to negative affect given a particular situation, whereas psychopathology questionnaire items are formulated in such a way that they assess how often symptoms are experienced (see Jorm et al., 1999). Second, BIS was also positively related to child-reported symptoms of Hyperactivity/Conduct problems and Aggression. This probably has to do with the fact that in children, externalizing symptoms are also thought to be BIS-mediated. For example, the influential temperament theory of Rothbart (see Rothbart \& Bates, 1998) posits that the temperamental factor of emotionality (or negative affectivity), which bears strong similarity to BIS, not only consists of fear and sadness, but also includes a lower-order trait of anger/frustration, which may contribute to aggressive behaviours when confronted with environmental stressors. Third, depression symptoms were not associated with lower BAS levels. It is most plausible that the non-clinical nature of the present sample accounted for this result, as previous studies with clinically referred patients have clearly demonstrated that lower BAS levels are connected to greater depression severity and less favourable clinical outcome (Kasch, Rottenberg, Arnow, \& Gotlib, 2002).

Admittedly, the current study suffers from a number of limitations. To begin with, it is important to note that the study was cross-sectional in nature. Thus, it should be kept in mind that it is not possible to draw conclusions on cause-effect relationships between BIS/BAS and psychopathological symptoms, and it is clear that longitudinal studies are needed in order to get insight into the exact nature of the relationships among these phenomena. Further, although correlations among variables were generally as hypothesized, some of the to be expected gender differences (e.g., girls scoring higher on Neuroticism, BIS, and symptoms of anxiety and depression) did not emerge, which indicates that the present sample was somewhat atypical. Another shortcoming has to do with the fact that some psychometric qualities of the BIS/BAS scales for children were not investigated. More precisely, the inclusion of a second BIS/BAS assessment and additional measures of negative affectivity and emotion regulation would have provided (further) information on the test-retest reliability and validity of the scales. Finally, although parent measures of children's psychopathological symptoms were included in the present study, the inclusion of parental scales of children's BIS/BAS (Blair, 2003) could have strengthened this study. In spite of these shortcomings, the study yields tentative support for the psychometrics of a BIS/BAS questionnaire for children, which seems to be a promising tool for researchers who are interested in studying these biological temperament factors in youths.

\section{References}

Blair, C. (2003). Behavioral inhibition and behavioral activation in young children: Relations with self-regulation and adaptation to preschool in children attending head start. Developmental Psychobiology, 42, 301-311.

Brown, K., Atkins, M. S., Osborne, M. L., \& Milnamow, M. (1996). A revised teacher rating scale for reactive and proactive aggression. Journal of Abnormal Child Psychology, 24, 473-480. 
Carver, C. S., \& White, T. L. (1994). Behavioral inhibition, behavioral activation, and affective responses to impending reward and punishment. Journal of Personality and Social Psychology, 67, 319-333.

Caseras, X., Avila, C., \& Torrubia, R. (2003). The measurement of individual differences in behavioural inhibition and behavioural activation systems: A comparison of personality scales. Personality and Individual Differences, 34, 999-1013.

Casey, B. J., Castellanos, F. X., Giedd, J. N., \& Marsh, W. L. (1997). Implication of right frontostriatal circuitry in response inhibition and attention-deficit/hyperactivity disorder. Journal of the American Academy of Child and Adolescent Psychiatry, 36, 374-383.

Chorpita, B. F., Yim, L., Moffitt, C., Umemoto, L. A., \& Francis, S. E. (2000). Assessment of symptoms of DSM-IV anxiety and depression in children: A revised child anxiety and depression scale. Behaviour Research and Therapy, $38,835-855$.

Depue, R. A., Krauss, S. P., \& Spoont, M. R. (1987). A two-dimensional threshold model o seasonal bipolar affective disorder. In . In D. Magnuson \& A. Öhman (Eds.), Psychopathology: An interactional perspective (pp. 95-123). San Diego, CA: Academic Press.

Dodge, K. A., Lochman, J. E., Harnish, J. D., Bates, J. E., \& Pettit, G. S. (1997). Reactive and proactive aggression in school children and psychiatrically impaired chronically assaultive youth. Journal of Abnormal Psychology, 106, $37-51$.

Eysenck, H. J. (1967). The biological basis of personality. Springfield, IL: Charles C. Thomas.

Eysenck, H. J., \& Eysenck, S. B. G. (1975). Manual of the Eysenck personality questionnaire (adult and junior). London: Hodder \& Stoughton.

Eysenck, S. B. G., Von Knorring, A., \& Von Knorring, L. (1988). A cross-cultural study of personality: Swedish and English children. Scandinavian Journal of Personality, 1, 215-221.

Fowles, D. C. (1980). The three arousal model: Implications of Gray's two-factor learning theory for heart rate, electrodermal activity, and psychopathy. Psychophysiology, 17, 87-104.

Franken, I. H. A., Muris, P. \& Rassin, E. (in press). Psychometric properties of the Dutch BIS/BAS scales. Journal of Psychopathology and Behavioral Assessment.

Goodman, R. (2001). Psychometric properties of the strengths and difficulties questionnaire. Journal of the American Academy of Child and Adolescent Psychiatry, 40, 1337-1345.

Gray, J. A. (1982). The neuropsychology of anxiety. New York: Oxford University Press.

Gray, J. A. (1987). The psychology of fear and stress. New York: Cambridge University Press.

Gray, J. A. (1991). The neuropsychology of temperament. In J. Strelau \& A. Angleiter. Explorations in temperament: International perspectives in theory and measurement. New York: Plenum.

Heubeck, B. G., Wilkinson, R. B., \& Cologon, J. (1998). A second look at Carver and White's (1994) BIS/BAS scales. Personality and Individual Differences, 25, 785-800.

Johnson, S. L., Turner, R. J., \& Iwata, N. (2003). BIS/BAS levels and psychiatric disorder: An epidemiological study. Journal of Psychopathology and Behavioral Assessment, 25, 25-36.

Jorm, A. F., Christensen, H., Henderson, A. S., Jacomb, P. A., Korten, A. E., \& Rodgers, B. (1999). Using the BIS/ BAS scales to measure behavioural inhibition and behavioural activation: Factor structure, validity, and norms in a large community sample. Personality and Individual Differences, 26, 49-58.

Kasch, K. L., Rottenberg, J., Arnow, B. A., \& Gotlib, I. H. (2002). Behavioral activation and inhibition systems and the severity and course of depression. Journal of Abnormal Psychology, 111, 589-597.

Lemery, K. S., Essex, M. J., \& Smider, N. A. (2002). Revealing the relation between temperament and behavior problem symptoms by eliminating measurement confounding: Expert ratings and factor analyses. Child Development, 73, 867-882.

Leone, L., Perugini, M., Bagozzi, R. P., Pierro, A., \& Mannetti, L. (2001). Construct validity and generalizability of the Carver-White behavioural inhibition/behavioural activation system scales. European Journal of Personality, 15, 373-390.

Matthys, W., Van Goozen, S., De Vries, H., Cohen-Kettenis, P. T., \& Van Engeland, H. (1998). The dominance of behavioural activation over behavioural inhibition in conduct disordered boys with and without attention deficit hyperactivity disorder. Journal of Child Psychology and Psychiatry, 39, 643-651. 
Muris, P., Meesters, C., \& Schouten, E. (2002). A brief questionnaire of DSM-IV defined anxiety and depression symptoms among children. Clinical Psychology and Psychotherapy, 9, 430-442.

Nigg, J. T. (2000). On inhibition/disinhibition in developmental psychopathology: Views from cognitive and personality psychology and a working inhibition taxonomy. Psychological Bulletin, 126, 220-246.

Quay, H. C. (1988). Attention deficit disorder and the behavioral inhibition system: The relevance of the neuropsychological theory of Jeffrey A. Gray. In L. M. Bloomingdale \& J. Sergeant. Attention deficit disorder: Criteria, cognition, and intervention. New York: Pergamon.

Quay, H. C. (1993). The psychobiology of undersocialized aggressive conduct disorder: A theoretical perspective. Development and Psychopathology, 5, 165-180.

Ross, S. R., Millis, S. R., Bonebright, T. L., \& Bailley, S. E. (2002). Confirmatory factor analysis of the behavioral inhibition and activation scales. Personality and Individual Differences, 33, 861-865.

Rothbart, M. K., \& Bates, J. E. (1998). In N. Eisenberg. Temperament \& Damon, W. (Eds.). Handbook of child psychology: Vol. 3. Social, emotional, and personality development (pp. 105-176). New York: Wiley.

Scheres, A., Oosterlaan, J., \& Sergeant, J. A. (2001). Response inhibition in children with DSM-IV subtypes of AD/HD and related disruptive disorders: The role of reward. Child Neuropsychology, 7, 172-189. 